\title{
Des soufis en banlieue parisienne
}

Mise en scène d'une spiritualité musulmane

\section{Mehdi Nabti}

\section{CpenEdition}

Journals

Édition électronique

URL : http://journals.openedition.org/assr/11593

DOI : $10.4000 /$ assr. 11593

ISSN : $1777-5825$

Éditeur

Éditions de l'EHESS

\section{Édition imprimée}

Date de publication : 1 décembre 2007

Pagination : 49-68

ISBN : 978-2-7132-2145-3

ISSN : 0335-5985

Référence électronique

Mehdi Nabti, « Des soufis en banlieue parisienne », Archives de sciences sociales des religions [En ligne], 140 | octobre - décembre 2007, mis en ligne le 02 juillet 2011, consulté le 19 avril 2019. URL : http:// journals.openedition.org/assr/11593; DOI : 10.4000/assr.11593 


\section{Mehdi Nabti}

\section{Des soufis en banlieue parisienne Mise en scène d'une spiritualité musulmane}

La zāwiya française de la Qâdiriyya-bûtchichiyya, une confrérie soufie issue du Maroc, connaît un développement transnational remarquable. Essor dû, dans les années 1970, à son leader actuel, Sîdî Hamza al-Qâdirî-Bûtchich ${ }^{1}$. À cette époque l'ordre religieux se développe sous une forme institutionnelle en Europe et principalement en France où la politique de regroupement familial (1974) favorise l'immigration maghrébine. Dès lors, des disciples plus ou moins actifs se réunissent régulièrement à domicile sous l'autorité d'un représentant du cheikh ${ }^{2}$. Il est difficile de cerner la singularité de cette zāwiya par rapport aux autres en terre d'immigration. Une telle démarche comparative manque actuellement d'enquêtes de terrain et de données empiriques concernant les différentes formes du soufisme contemporain dans les sociétés occidentales ${ }^{3}$. Les travaux de recherche

1. Né en 1922 à Madagh au nord-est du Maroc. Au début des années 1970, Hamza, alors disciple de Shaykh 'Abbas Qâdirî-Bûtchich (1890-1972), fut en concurrence avec 'Abdesslam Yassine pour la direction de l'ordre qâdirî-bûtchichî. Pour des raisons de lignage familial, Yassine fut évincé au bénéfice de Hamza. Il fonda ensuite son propre parti politique Al-'adl wa al-ihsane (Justice et Bienfaisance). Les rapports entre la Qâdiriyya-bûtchichiyya et Al-'adl wa al-ihsane on été étudiés de près par 'Abdelilah Bouasria (2007).

2. D'après les données recueillies durant cette enquête, il semble que ce sont les immigrés marocains qui ont introduit la doctrine qâdiriyya-bûtchichiyya en France dans les années 1970. Certains sont toujours présents en région parisienne, mais, ceux-ci ayant refusés de participer aux entretiens, je n'ai pu en savoir davantage sur les motifs de cet essaimage confrérique et les modalités d'organisation de l'ordre religieux à cette époque.

3. C'est l'une des raisons pour lesquelles avec Mouloud Haddad (doctorant EHESS), nous avons créé en 2003 l'association Pour Une Recherche sur l'Islam et les Sociétés du Monde Musulman (PRISMM). À cette époque, M. Haddad et moi-même avons eu l'idée d'organiser un atelier doctoral pluridisciplinaire à l'EHESS autour des pratiques contemporaines du soufisme en Occident. Après rédaction d'un appel d'offre, nous avons sollicité Hasssan Elboudrari comme tuteur scientifique. L'atelier doctoral, ouvert au public, s'est tenu début juin 2004 dans les locaux de l'EHESS. L'un des objectifs de cette journée d'étude, mêlant des doctorants venus d'horizons institutionnels et disciplinaires divers, a donc été de montrer que le soufisme européen correspond à une tradition et à une pratique vivantes, que son implantation résulte d'une histoire encore à écrire et qu'il fait partie, en tant que mouvement de fond, des questions que soulève l'intégration de l'islam et des communautés musulmanes dans les sociétés occidentales, au même titre que d'autres mouvements, laïques ou fondamentalistes, qui les traversent. 
sur l'islam des vingt dernières années ont privilégié l'analyse politique au détriment de l'approche anthropologique et sociologique. Si les rapports entre religion et politique sont une réalité indiscutable de l'islam contemporain, son étude ne nous informe en rien sur la place de cette religion dans la vie quotidienne. Jusqu'à très récemment, les chercheurs en sciences sociales européens considéraient l'islam comme un simple fait migratoire, dit de " transplantation ${ }^{4}$ : l'islam désormais implanté en Europe a fait naître une nouvelle approche scientifique, celle des conversions. Face à un phénomène aussi complexe que les ordres mysticoreligieux musulmans, notre but est de saisir les modes de fonctionnement d'une branche d'adeptes de la Qâdiriyya-bûtchichiyya en répondant aux questionnements suivants : 1 . Pourquoi et comment une zāwiya maghrébine s'implantet-elle en France ? 2. Quelles en sont les activités ? Comment est-elle organisée? 3. Qui sont les disciples ? Peut-on en établir un profil type ? 4. Pourquoi des individus choisissent-ils de s'affilier à une confrérie soufie maghrébine dans une société laïque de tradition catholique ? Comment se met en scène sa liturgie rituelle? Dans quel but?

On tentera de répondre à ces questions en trois points : la description physique de la zāwiya et de son organisation interne ; l'étude des différentes activités qui s'y déroulent; la mise à jour du rituel et de sa mise en scène.

Mais pourquoi avoir choisi d'étudier la Qâdiriyya-bûtchichiyya ? Quelles en sont les particularités?

\section{La Qâdiriyya-bûtchichiyya}

Par le nombre très important d'intellectuels, d'artistes, de personnages publics et d'hommes politiques qui en font partie, la confrérie Qâdiriyya-bûtchichiyya bénéficie d'une force de lobbying telle que son crédit moral et la puissance de ses réseaux dépassent de loin ceux de la totalité des ordres mystiques maghrébins. Les sources bio-hagiographiques privées fournies par les dirigeants lors de notre enquête font remonter la généalogie de Sîdî Hamza jusqu'au Prophète par l'intermédiaire de 'Abd al-Qâdir al-Jîlânî. La confrérie Qâdiriyya-bûtchichiyya est présente dans presque tous les pays où vivent des musulmans, avec un nombre particulièrement élevé de disciples en France, aux États-Unis, au Canada, en Finlande, en Allemagne et en Angleterre. Trois raisons expliquent, selon nous, ce capital de sympathie : 1 . Les dirigeants de la confrérie consacrent énormément d'énergie à médiatiser le message de leur cheikh à l'aide de sites Internet, festivals de musique, séminaires, revues, publications littéraires et conférences assurées parfois par Faouzi Skali ${ }^{5}$ ou des disciples convertis. Leurs stratégies de recrutement

4. Ce terme a été employé, dès 1984, par Dassetto et Bastener dans leur ouvrage L'islam transplanté. Vie et organisation des minorités musulmanes de Belgique.

5. Faouzi Skali est un disciple et l'un des représentants de la confrérie. Auteur de plusieurs ouvrages, articles et interviews sur le soufisme, il a été pendant plusieurs années le directeur du 
les ont amenés à supprimer le cheminement initiatique propre au soufisme traditionnel : " maintenant c'est la confrérie qui cherche le disciple ${ }^{6} ; 2$. La confrérie accueille des femmes et aime faire la promotion de l'égalité sexuelle. Cependant, cette affirmation est à nuancer car les espaces d'invocations ne sont pas mixtes et les dirigeants sont tous des hommes issus de la lignée du fondateur ; 3. Son leader actuel, Sîdî Hamza, est vu par ses disciples comme un héritier du « secret initiatique" (sirr) et le "pôle spirituel» (qutb). Ce fait est très important car l'autorité charismatique qu'il exerce sur ses disciples du monde entier est exceptionnelle. Diverses études ont prouvé que les adeptes remettent souvent en cause le pouvoir symbolique exercé par le successeur d'un cheikh qui devient peu à peu un simple héritier "gestionnaire du charisme ${ }^{7}$.

Les écrits antérieurs consacrés à cette confrérie se sont surtout intéressés à la bio-hagiographie du cheikh actuel tout en spécifiant les filiations doctrinaires et les caractéristiques ésotériques de son enseignement spirituel ${ }^{8}$. Certains journalistes et sociologues ont récemment révélé les rapports de connivence que le cheikh et ses subalternes entretiennent avec le Palais royal dans un Maroc où l'islamisme politique connaît une audience considérable dans les couches moyennes et éduquées de la population ${ }^{9}$. Pour toutes ces raisons, il nous a semblé intéressant de s'interroger sur les modalités d'inscription de cette confrérie en France et plus précisément en région parisienne. Avant d'aborder le vif du sujet, définissons le cadre théorique de cette enquête.

Festival des Musiques Sacrées de Fès. En 2007, il a fondé dans cette même ville un festival concurrent, le Festival de Fès de la Culture soufie et du Développement humain. Lors des colloques et concerts qu'il organise, Skali met un point d'honneur à promouvoir sa confrérie en invitant musiciens, chanteurs, convertis et disciples universitaires affiliés au Shaykh Hamza, sans oublier les chercheurs occidentaux qui se consacrent au sujet. J'ai abordé la problématique des stratégies corporatistes du soufisme maghrébin et des conflits internes qu'elle engendre dans une communication orale intitulée "La visibilité publique des confréries soufies en France et au Maroc : enjeux politiques, stratégies individuelles et conséquences internes d'un nouveau modèle religieux" (Paris, EHESS, juin 2007, Journée d'études Privé et public dans l'islam contemporain, Cadis).

6. L'un des slogans favoris des représentants et partisans de l'ordre religieux.

7. La notion de "gestionnaire du charisme " a été théorisée par H. Elboudrari (1985) en se basant sur les modèles de $\mathrm{M}$. Weber. Cette idée a été discutée et affinée à propos de l'organisation interne de la confrérie Aissâwiyya au Maroc (Nabti, 2007 : 245 sq.)

8. Sur l'historique officiel de la confrérie et ses rattachements doctrinaires, voir Karim Ben Driss, auteur d'un texte hagiographique adoubé par les dirigeants de la confrérie (2002).

9. Ce fait s'intègre dans les stratégies de soumission des confréries soufies par l'État marocain. Depuis son arrivée au pouvoir, la dynastie alaouite sauve et maintient le capital symbolique des confréries à l'aide de différentes stratégies d'assujettissement - entre autres, la mise en tourisme des aspects artistiques du mysticisme et de la fonctionnarisation des hauts responsables des ordres religieux. En contrepartie, les descendants des saints et les cheikhs doivent afficher une opinion politique complaisante: voir Nabti, 2007: 38-68. En outre, les relations entre l'État marocain, l'islamisme politique et la confrérie qâdiriyya-bûtchichiyya ont été étudiés par Bouasria, 2007 ; Dialmy, 2000 : 05-27 ; Agnouche, 1987 ; Kadiri, 2002 ; Amrous, 1986. 


\section{Cadre théorique et méthodologie}

La connaissance scientifique et principalement anthropologique sur le mysticisme maghrébin est très largement théorisée et riche en analyses explicatives. Ce savoir a évolué depuis la seconde moitié $\mathrm{du} \mathrm{XX}^{\mathrm{e}}$ siècle sous l'impulsion de l'anthropologie anglo-saxonne menée par des chercheurs américains au Maroc comme Dale Eickelman (1981), Clifford Geertz (1973, 1992 [1968], 2002 [1983]), Ernest Gellner (1981, 2003 [1969]), Spencer Trimingham (1971) et Vincent Crapanzano (2000 [1973]). À la même période, les français Émile Dermenghem (1982 [1954]) et Jacques Berque (1955) ont offert des modèles théoriques pertinents pour l'étude des pratiques religieuses musulmanes. Ainsi, la notion d' "islam local » (approfondi notamment par Geertz et Trimingham) développée par Berque dans son ouvrage Les structures sociales du haut Atlas (1955) permet d'aborder l'islam comme un système composite vécu. Ce principe remet en question l'idée de l'immuabilité des standards religieux tout en dépassant le modèle segmentant de Gellner qui distingue "islam rural vs islam urbain » ou "islam savant $v s$ islam populaire ». Dans le prolongement des idées de Berque, nous avons choisi d'employer ici un cadre microsociologique en référence au modèle goffmanien. Dans son ouvrage intitulé La mise en scène de la vie quotidienne, Ervin Goffman (1973) pose les bases de la microsociologie en envisageant la vie comme un théâtre, avec ses acteurs et ses scènes où se déroulent des « rites d'interaction ». Le terme d'interaction suggère, dans son étymologie même, l'idée d'une action mutuelle, en réciprocité. Appliquée aux relations humaines, cette notion implique de considérer la communication comme un processus d'interaction sociale où des règles théoriques et des dispositifs visuels fondamentaux règlent et ordonnent les relations entre les personnes. Ce qui nous intéresse dans les notions de Goffman, et que nous pouvons utiliser dans le cas d'une zāwiya soufie, c'est ce qui s'applique à la communication corporelle et visuelle non verbale. Ce cadre de référence se révèle approprié à l'étude des systèmes rituels car il permet de décrire et de comprendre le rôle joué, d'une part, par l'aménagement de l'intérieur d'un sanctuaire, et, d'autre part, par chacun des protagonistes qui s'y trouvent.

Notre méthodologie combine à la fois l'observation participante, les entretiens libres et semi-directifs appliqués à une enquête de terrain en 2004 à l'intérieur de l'une des zāwiya-s masculines de la confrérie qâdiriyya-bûtchichiyya. Par leur aspect hétérogène, certaines caractéristiques de notre échantillon (quarante-sept personnes) sont précieuses pour définir le modèle confrérique implanté dans une société laïque de tradition catholique. Ces caractéristiques sont les suivantes: l'échantillon est masculin ${ }^{10} ; 30 \%$ des disciples sont des convertis ${ }^{11}, 60 \%$ sont

10. À l'époque de cette étude, les femmes affiliées ne disposent pas de zāwiya et se réunissent sous l'autorité de deux responsables féminines au domicile de différentes adeptes (Paris intramuros et petite couronne). Ces lieux de réunions, de fréquence hebdomadaire et géographiquement rotatifs, sont restés inaccessibles à cause de la non-mixité des espaces d'invocations de la tarīqa.

11. Cette donnée de l'échantillon contredit l'affirmation de Éric Geoffroy qui écrit que «la Butchîchiyya a opté pour la séparation entre disciples d'origine marocaine et disciples de souche 
des Marocains récemment immigrés (pour études, travail ou dans le cadre du regroupement familial) et seulement $10 \%$ sont des Français d'origine algérienne (plus précisément d'ascendance kabyle) qui ne parlent pas l'arabe.

L'échantillon est recruté en milieu urbain : $100 \%$ des enquêtés vivent en banlieue parisienne ; l'échantillon est en majorité composé de personnes bilingues arabe/français $(65 \%) ; 76 \%$ des disciples sont âgés de 20 à 35 ans ; $67 \%$ ont des diplômes universitaires de second cycle. $38 \%$ ont des diplômes d'ingénieurs ou équivalent et $15 \%$ possèdent un doctorat ; $68 \%$ n'ont pas d'emploi formel, habitent chez leurs parents et sont célibataires ; $69 \%$ ont un emploi dans le secteur des services, de la vente et de la restauration rapide ; $31 \%$ sont des cadres ou ingénieurs ; $40 \%$ ont découvert la religion musulmane par le biais de la confrérie.

Le profil social des disciples enquêtés dans la zāwiya qâdiriyya-bûtchichiyya est extrêmement varié. On y rencontre beaucoup de personnes atypiques ou ayant un parcours complexe. Dans la perception populaire française, " arabe " et "musulman"sont souvent considérés comme synonymes. Mais les Arabes présents en France peuvent aussi être chrétiens ou agnostiques, alors que des musulmans français peuvent avoir des origines non arabes : convertis, Turcs, Berbères, Iraniens, Kurdes, Maliens, Sénégalais, etc. De fait, le nombre de convertis à l'islam est particulièrement important parmi les disciples sondés, mais tous sont les premiers destinataires de l'enseignement doctrinal du cheikh marocain. L'échantillon testé ici ne reflète pas l'exhaustivité de l'effectif des partisans de Sîdî Hamza en banlieue parisienne, car des centaines d'affiliés vivent leur spiritualité dans l'intimité de leur domicile et isolés du groupe étudié. Mais pour les individus enquêtés comme pour tous les aspirants au cheminement initiatique, la pratique du soufisme nécessite de respecter deux types d'impératifs : les rites obligatoires imposés à tous les musulmans (le chemin de la Loi, al-sharī'a) et les rites surérogatoires à destination des seuls initiés enseignés par des maîtres (shuyūkh) au sein d'une confrérie où se transmet une doctrine, "la voie étroite " (al-tarīqa). Les enseignements mystiques englobent, d'une part, des recommandations morales/éthiques, et, d'autre part, des récitations de litanies spirituelles, $d h i k r$, wird et hizb - censées permettre au disciple de vivre l'expérimentation de l'union avec Dieu au cours de la vie terrestre. Il s'agit d'atteindre, par étapes successives, celle de l'anéantissement de son être dans l'unicité divine (al-fanā' fī al-tawhīd), manifestant ainsi sa rencontre avec la vérité (al-haqq). Certaines confréries autorisent la mise en scène de pratiques artistiques et musicales telles que l'écoute $\left(a l\right.$-sam $\left.\bar{a}^{\top}\right)$ de chants liturgiques et les danses de la présence (al-hadra) provoquant chez les fidèles des phénomènes d'extase $(a l-h \bar{a} l)$ et de transe. C'est principalement dans les zāwiya-s que se pratique cette approche de la spiritualité.

européenne ». Cette observation est surprenante, car je n'ai jamais pu constater un tel phénomène de distinction spacio-ethnique au sein de cet ordre, ni en France ni au Maroc. Malheureusement Geoffroy n'indique pas où et quand il a observé ce clivage (Le soufisme et la France (partie 2/2), publié sur www.oumma.com, le vendredi 29 juin 2007). 
Située à $30 \mathrm{~km}$ de Paris dans un quartier résidentiel assez facilement accessible en transport en commun, la zāwiya qâdiriyya-bûtchichiyya se trouve au rez-dechaussée d'un pavillon de banlieue d'une superficie d'environ $70 \mathrm{~m}^{2}$. Louée depuis la fin des années $1990^{12}$ par quatre jeunes disciples (étudiants et salariés), son apparence extérieure est très banale. C'est pourtant là que se retrouvent à la fois le faqìr habitué des lieux ou le nouvel affilié qui souhaite vivre son mysticisme.

\section{Hiérarchie interne}

La hiérarchie interne se compose de trois niveaux. Pour encadrer en ces lieux le commun des fuqarā' (plur. de faqìr), un responsable (le muqaddem) est aidé dans sa tâche par quelques fuqarā'-adjoints.

Bien qu'il n’y réside pas, le muqaddem (= délégué) est le seul et véritable chef de la zāwiya. Trentenaire et docteur en sciences des religions, il est issu du lignage du fondateur et nommé à la direction du sanctuaire par le cheikh en personne ${ }^{13}$. Il s'y rend plusieurs fois par semaine car ses responsabilités sont multiples : il est chargé de surveiller et de diriger les différentes activités liturgiques, mais aussi de clarifier les paroles du maître résident au Maroc, de donner le pacte au nouvel affilié, de diriger les séances d'invocations collectives, de gérer la communication publique de la confrérie. Toujours très attentif, il veille aux relations que les disciples entretiennent les uns avec les autres et consacre ainsi beaucoup de temps à les écouter et s'investit personnellement lorsque ceux-ci le sollicitent (bénédiction d'un nouveau-né, d'un foyer, de la réussite scolaire ou professionnelle, aide à la guérison, etc.). Représentant officiel du cheikh en France et en Europe, il est censé bénéficier d'une part importante de sa bénédiction (baraka) et sait faire preuve de beaucoup d'affabilité. Côté pédagogique, le muqaddem met l'accent sur le respect absolu de la religion canonique en clarifiant les notions de base $\mathrm{du}$ soufisme maghrébin. Il surveille de près l'élaboration de documents audiovisuels ${ }^{14}$,

12. Il en existe une antérieure, ouverte dans les années 1970 par des immigrés marocains, que je n'ai pu visiter.

13. Ici, et comme dans la grande majorité des confréries mystiques africaines, la succession fut donnée d'abord aux fils biologiques sur les disciples. Ce fait découle de la problématique de la chefferie tribale en milieu confrérique. Voir Hammoudi, 2001 ; Boubrik, 2000 ; Elboudrari, 1985 ; Hamès, 1991 : 99-118. Pour une réflexion anthropologique sur les alliances matrimoniales et tribales liées aux stratégies de successions héréditaires, voir Bonte, 2001.

14. Les disciples de la confrérie réalisent des DVD artisanaux commercialisés dans les salons de rencontres d'associations musulmanes. On y voit l'historique de la tarìqa, illustré par de courtes vidéos des rencontres avec Sîdî Hamza, des séances d'invocations collectives, des chants religieux et des témoignages de disciples. L'accent est mis sur les Européens convertis et les jeunes femmes. La volonté affichée et clairement déclarée est, d'une part, de faire de la publicité à l'ordre religieux, et, d'autre part, de "donner une bonne image de l'islam », comme nous l'ont souvent affirmé les disciples interrogés. 
dirige la maintenance du site Internet ${ }^{15}$ de la confrérie et organise avec un groupe réduit de fuqarā'-adjoints des concerts et des conférences-débats. Interrogé sur son propre rôle, il s'efface devant le discours officiel de sa confrérie :

La tarīqa Qâdirîyya-Bûtchichiyya est une voie d'amour. Ici nous ne faisons pas de politique, nous ne sommes pas là pour aimer les musulmans ou seulement les soufis. Nous sommes là pour tenter d'apaiser les cœurs. Il faut aimer toutes les créatures vivantes, car tout est création divine.

Étant un descendant direct du cheikh, le muqaddem bénéficie auprès des disciples d'un fort capital de sympathie - voire d'adoration - qui justifie pour certains leur présence au sein de la zāwiya. Les maîtres fondateurs des confréries sont considérés par les fidèles, après leur mort ou même de leur vivant, comme de véritables saints investis de pouvoirs extraordinaires. À ce propos, E., 27 ans, étudiant en DEA de physique à Paris, nous dit :

Je faisais des ijtima $\bar{a}^{-}$-s (réunions de disciples) avec mon père à Casablanca, avant de venir en France poursuivre mes études, mais l'ambiance était très différente, juste " bonjour, bonsoir ». C'était bien mais ici c'est vraiment exceptionnel. Grâce au muqaddem, il y a une forte présence divine et beaucoup d'amour entre les fuqarä'. Le muqaddem m'interroge souvent au sujet de mes études, il est très attentif et me dit de ne pas baisser les bras, de m'accrocher à mes études et au soufisme, car la méthode spirituelle stabilise le nafs. Le muqaddem sait tout mieux que nous, il est divin.

Autour du muqaddem et proche de lui (au sens propre comme au figuré), se trouve un groupe d'une demi-douzaine de fuqarā' que, faute de désignation précise, on peut nommer les fuqarā'-adjoints. Parmi eux, des " anciens ", à savoir des fuqarā' marocains vivant en France, tous retraités. Ils ont une connaissance parfaite des invocations de la tarìqa et bénéficient d'une solide formation religieuse leur permettant de réciter de nombreuses sourates du Coran par cœur. Ils sont en très petit nombre dans la zāwiya mais bénéficient de beaucoup de respect. Ils n'ont pas de rôle défini mais leur présence et leur expérience permet le bon déroulement des invocations collectives. À côté des "anciens ", il y a les jeunes et quelques Marocains de haut niveau social (ingénieurs ou chefs d'entreprise) ayant pris connaissance de la voie dans leur jeunesse ou par tradition familiale, qui sont souvent des amis intimes du muqaddem. Il est intéressant de signaler la présence de fuqarä'-adjoints convertis, très jeunes (25 ans en moyenne). La majorité sont des étudiants non arabophones et jouissent d'un important capital de sympathie auprès du muqaddem et des " anciens ". Le rôle des jeunes est

15. www.saveurs-soufies.com : il s'agit du site officiel de la confrérie en Europe, réalisé par les disciples dans cette zāwiya. Très visité, ce site est à but pédagogique et traite des principes de l'islam, de l'histoire du soufisme et de la confrérie. Le site permet aussi de participer à un forum de discussion et d'écouter des chants religieux et des extraits du Coran sur mp3. Il existe aussi un Intranet privé réservé aux fuqarā' qui leur permet de se contacter et d'échanger sur divers sujets. Cet Intranet contient les invocations individuelles et collectives enseignées à la zāwiya, l'agenda des activités, les principes de base du soufisme et du disciple, des vidéos et des photographies, des supports de cours et des fiches de lecture. 
principalement d'organiser et d'administrer les séances d'invocations collectives (distribution des livrets des invocations et des prières qu'ils ont eux-mêmes écrits en phonétique et en arabe) et de remplacer le muqaddem, le cas échéant. Ce sont eux qui organisent les fréquents voyages à la zāwiya-mère au Maroc, pour permettre à une majorité de fuqarā' de rencontrer le shaykh lors d'une visite (al-ziyāra) estivale annuelle. Par leur disponibilité et leurs compétences organisationnelles et techniques, ils participent pleinement à la vie privée et publique de la zāwiya.

Le commun des fuqarā' présent à la zāwiya ne fait pas partie de l'entourage proche du muqaddem. Ils s'organisent pour assurer l'intendance de la zāwiya (mise en relation des fuqarā', gestion du budget, préparation des repas collectifs, organisation des tâches ménagères). Leur moyenne d'âge est d'environ trente ans et un tiers sont des convertis par l'intermédiaire de la Voie (tarīqa). La rencontre du faqìr avec la tarīqa se fait généralement de façon informelle, par le biais d'amis, de la famille, de collègues ou même de façon fortuite, parfois, lors de recherches personnelles sur Internet. Dans tous les cas, la personne est invitée à une séance de $d h i k r$ au domicile d'un faqìr et, si elle le désire, elle se rendra ensuite à la zāwiya pour rencontrer le muqaddem. Attirés au départ par une recherche spirituelle personnelle, le côté ésotérique de l'islam a motivé les fuqarā' pour vivre ce cheminement initiatique. Face aux convertis présents dans la zāwiya, on a affaire à un discours caractéristique (indépendamment de l'islam, du soufisme, de la zāwiya en question) qui opère une césure radicale entre un avant " dissolu » et un après plein d'espoir et d'intégrité. Voici, par exemple, le témoignage de S., 31 ans, cadre dans l'édition, qui a trouvé dans cette pratique la finalité de ses recherches personnelles :

Auparavant j'étais catholique pratiquant, mais tout cela était très moraliste. Il y a dix ans, j'étais à fond dans le bouddhisme, petit véhicule et tout. Mais un jour je suis tombé sur un ouvrage de René Guénon ${ }^{16}$, j'ai acheté tous ses livres et j'ai vraiment accroché. J'ai voulu vivre ce dont il parlait, la transcendance et le parcours initiatique. Je cherchais parallèlement quelque chose qui pouvait me renforcer dans ma foi, comme une pratique supplémentaire. J'ai cherché à droite, à gauche et j'ai lu récemment le " livre des haltes » de l'émir 'Abd al-Qâder ${ }^{17}$ et je me suis rendu compte qu'il avait vraiment goûté quelque chose d'exceptionnel dans le soufisme, ce quelque chose que je recherchais. J'ai ensuite fait des recherches sur Internet et je suis tombé sur le site "saveurs soufies », j’ai envoyé un email et maintenant je suis là ! (...) Avant de me convertir à l'islam et d'être dans la voie, j'avais une vie complètement décousue. Je ne voulais pas travailler, je me laissais complètement aller, tout autour de moi me semblait incohérent. Ma vie elle-même était en fait incohérente. La voie m'a apporté beaucoup de bienfaits, comme la discipline. Maintenant je suis stabilisé, j'ai un bon travail et tout dans ma vie s'est sacralisé.

16. René Guénon (1886-1951), auteur et métaphysicien français qui a fait connaître en Occident les philosophies orientales.

17. L'émir Abd al-Qâdir (1808-1883), personnage historique devenu légendaire, fut à la fois écrivain, poète, mystique, philosophe, résistant militaire et le fondateur de l'État algérien. 
Ce genre de témoignage entraîne instantanément une foule de questions (sur la conversion à l'islam par le biais du soufisme, sur les relations entre les convertis et les Français d'origine maghrébine présents dans la confrérie par tradition familiale, sur les musulmans qui vivent un " renouveau " religieux, sur le rapport à la langue arabe, aux hiérarchies) qui dépassent largement le cadre de la présente étude. Tous les fuqarā' interrogés s'accordent pour évoquer les liens intimes qui les unissent ; le vocabulaire qu'ils emploient s'inscrit dans différents champs sémantiques (amour et amitié désintéressée entre fuqarā' de différents niveaux sociaux et de différentes cultures, sérénité, patience, générosité, humilité, discipline, connaissances coraniques, politesse, respect, conseils, etc.). Ils sont les premiers destinataires des activités de la zāwiya, de la doctrine et du discours du cheikh qui semble leur apporter de nombreux bienfaits. C'est ce que nous dit M., 31 ans, pharmacien d'origine algérienne : la pratique lui a permis de rencontrer l'émotion spirituelle :

Au fur et à mesure des années, ma pratique religieuse est devenue "froide » : je priais

de façon mécanique, sans émotion. J'ai retrouvé, grâce à la confrérie, une spiritualité qui m’avait échappé.

Parmi le commun des fuqarā', se trouve le groupe de chant liturgique $\left(s a m \bar{a}^{\prime}\right)$ : de jeunes Marocains (quatre à huit personnes, ingénieurs et étudiants), possédant à la fois une belle voix et une bonne connaissance des chants religieux maghrébins (qasida). Groupe vocal sans accompagnement instrumental, puisant son répertoire dans le malhūn (poésie chantée de tradition citadine marocaine) et dans la burda ${ }^{18}$, il se produit régulièrement en concert en région parisienne ou en Belgique et participe à toutes les veillées spirituelles de la confrérie.

Après la hiérarchie interne de la zāwiya, on peut observer les différentes activités qui s'y déploient. Les personnes rencontrées affirment que les activités de la zāwiya française sont calquées sur celles de la zāwiya-mère marocaine. Il faudrait le vérifier, mais l'analyse de leurs attitudes permet de déceler, chez eux, un certain sentiment de honte de l'intimité.

\section{Les activités de la zāwiya, une honte de l'intimité ?}

Les activités internes concernent l'organisation de l'aspect éducatif, spirituel et cultuel que la zāwiya offre à tous les fuqarā': l'enseignement et la pratique spirituelle propre à la tarīqa, établie et autorisée par le cheikh. Depuis le Maroc, celui-ci a instauré un certain nombre de règles concernant des comportements idéaux et des pratiques religieuses précises actuellement implantées en région parisienne. Chaque jour de la semaine, une activité précise est dispensée par le muqaddem aidé des fuqarā'-adjoints. Les activités privées principales de la zāwiya sont les ijtimáa ${ }^{c}$-s, réunions, qui se déroulent à partir de la prière du coucher du soleil (maghrib) et qui peuvent se poursuivre jusqu'au petit matin.

18. Al-Burda (litt. "le manteau »), ensemble de poèmes d'Al-Bûsîrî (XIII siècle) consacré à l'éloge du Prophète. 
Ces ijtima $\bar{a}^{\text {'s }}$ ont lieu dans l'espace principal et secondaire (voir plan) et permettent aux fuqarā' de réciter les invocations sonores et collectives. Le Coran est lu collectivement et silencieusement, dans sa totalité, par les fuqarā’ tous les samedis soirs, lors d'une très longue soirée où des chants spirituels (samā') acheminent vers la transe de la Présence (al-hadra) qui provoque des mouvements corporels, des cris et des pleurs chez les disciples. Lors de cette soirée, les enseignements (dars, pl. durūs) revêtent une grande importance. Réalisés à tour de rôle par deux fuqarā', ils ont pour sujet le droit musulman (fiqh), l'explication de hadiths (dires du Prophète) et des aperçus de la vie du Prophète. À tour de rôle, les adeptes préparent une recherche qu'ils exposent oralement devant l'assistance sous l'écoute attentive du muqaddem. Celui-ci clôt le compte rendu par un résumé à connotation spirituelle, en insistant sur quelques points précis. Cette soirée se termine par un grand repas collectif préparé pendant de longues heures dans la cuisine par deux fuqarä' (à tour de rôle, ici aussi, ce ne sont jamais les mêmes qui préparent les repas) qui ne participent pas de ce fait aux invocations collectives. Le dimanche matin, un petit déjeuner est offert à ceux qui ont passé la nuit sur place car certains fuqarā' se déplacent de très loin (lointaine banlieue, Angleterre, Belgique) et la zāwiya leur offre aussi le gîte. Il faut ajouter à ces activités hebdomadaires les voyages à destination de la zâwiya-mère au Maroc, organisés plusieurs fois par an par les fuqarā'-adjoints, au rythme des fêtes musulmanes (Ramadan, Ä̈d, etc.) ainsi que pour la retraite spirituelle (al-khalwa) estivale, moment important qui permet à des milliers de disciples venus du monde entier de rendre une visite (al-ziyāra) au cheikh.

Les activités externes ont trait à l'organisation d'activités culturelles ouvertes aux non affiliés, encadrées et animées par les fuqarä, adjoints ou non, au sein d'associations culturelles à but non lucratif (dites de « loi 1901 »). Par ce biais, la zāwiya offre diverses activités sportives (football), artistiques (apprentissage de chants spirituels, de poésie, des cours de théâtre) et éducatives (soutien scolaire, alphabétisation en français et cours d'arabe classique, individuels, à la demande). L'investissement de l'espace public prend aussi la forme de concerts de chants spirituels par les disciples, de conférences-débats sur le soufisme par diverses associations culturelles, de publications, de vente de DVD artisanaux et du site Internet de la confrérie. On constate que les activités publiques de la zāwiya sont immanquablement orientées vers la communication d'une promotion intellectuelle, sportive et culturelle du mysticisme où la transe et les longues invocations surérogatoires quotidiennes sont volontairement passées sous silence. Pourquoi les hauts responsables de cet ordre ne promeuvent-ils pas les pratiques plébiscitées par les disciples? Il est clair que les effets spectaculaires (cris, danses, pleurs) manifestés lors de certaines réunions d'adeptes auxquelles nous avons pu assister ne reflètent pas, pour les dirigeants, l'image du "soufisme authentique " (selon leur propre expression), bien qu'ils soient le fait d'un grand nombre d'adeptes, convertis ou non. La gestion publique par les autorités de cette zāwiya (et par extension de l'ordre religieux lui-même en France et en Europe) dévoile ainsi un sentiment de honte de l'intimité. Des secrets inavouables sont, pour reprendre une expression de Goffman, "incompatibles avec l'image que le représentant de 
l'autorité s'efforce de maintenir en public»(Goffman, 1973 : 137). Interdire ces manifestations apparaît comme impossible, car elles sont paradoxalement plébiscitées par les dirigeants qui y voient une forme de bénédiction (baraka) divine justifiant l'allégeance des disciples à leur cheikh. Mais le muqaddem montre qu'il maîtrise ces formes religieuses extatiques et qu'il a prise sur elles par son intervention sur le déroulement de toutes les activités de la zāwiya.

\section{Le territoire du divin}

Aborder la problématique de la présence du divin dans un espace intérieur c'est tout d'abord s'interroger sur les notions fondamentales de sacré et de profane, sachant qu'en islam le sacré est, avant tout, dominé par les notions de licite et d'illicite. La religion musulmane étant fondée sur la distinction entre halāl (permis) et harām (interdit), la langue arabe possède un champ sémantique qui manifeste cette idée, tel que la sanctification (al-muqaddas), la délimitation du périmètre saint (al-hurm, "le refuge ») et la pureté (al-quds). Mais, dans le cas d'une zāwiya, les choses se compliquent quelque peu car la séparation entre le licite et l'illicite n'est pas clairement tranchée, du moins au niveau des actes de piétés accomplis par les visiteurs. Dans Les formes élémentaires de la vie religieuse (Durkheim, 1998 [1912]), le fondateur de la sociologie française nous informe que la religion peut être définie comme un ensemble de croyances et de rites portant sur des objets ou des domaines sacrés. Et le sacré, c'est ce qui se distingue du profane, cette distinction étant le minimum nécessaire à la pensée pour établir une première classification et s'affirmer ainsi comme pensée sociale. Si cette distinction existe au niveau de la pensée, il faut découvrir comment elle s'exprime au niveau de la pratique sociale. La routinisation du charisme du cheikh permet la fixation dans la vie sociale, et même dans les accessoires matériels, de la croyance en la manifestation du divin. Il faut donc qu'il y ait un lieu précis où se manifeste le sacré par distinction avec le profane. Ce lieu est celui au-delà duquel le profane peut exister et concentre vers lui toute l'activité sociale des croyants. Selon Durkheim, pour qu'il y ait religion, il faut qu'il y ait distinction spatiale entre le lieu du sacré, l'endroit qui accueille la manifestation de la piété sociale à travers le culte, et le lieu du profane, où les activités domestiques ordinaires se déroulent.

L'intérieur de la zāwiya (murs peints en blanc, fine moquette de couleur vert foncé) est d'une sobriété proche de l'austérité : elle est dépourvue de meubles mais équipée d'un grand canapé, d'un téléviseur et de quelques gros coussins disposés le long des murs de l'espace d'invocation principal. L'espace multimédia bénéficie d'une grande bibliothèque et d'un bureau sur lequel sont disposés trois ordinateurs. Si aucun élément décoratif ne vient divertir l'œil du faqīr, la zāwiya accueille néanmoins des actes rituels et des dispositifs visuels qui ont pour fonction de manifester l'appartenance du lieu à la tarīqa. Ils prennent place au sein de deux espaces distincts qu'on peut nommer espace rituel et espace profane.

L'espace rituel est l'endroit où se manifeste la présence extraordinaire du sacré. Il s'agit de «l'arrière-scène » (Goffman, 1973 : 105 sq.) de la représentation constituée ici des espaces d'invocations, de l'espace multimédia et de la bibliothèque 
(endroit où s'isole le muqaddem et où sont conservés les recueils d'invocations et les exemplaires du Coran). L'espace rituel accueille la totalité des pratiques rituelles qui rythment la vie quotidienne de la demeure. Il est fermement interdit aux disciples d'être pieds nus dans l'espace rituel, sous peine d'y ramener des impuretés. Des sandales en plastique sont à leur disposition et leur permettent de passer d'un espace à l'autre ; celles-ci doivent impérativement rester dans l'espace profane.

C'est le groupe de chant liturgique qui permet de passer d'une ambiance de recueillement, lors des invocations collectives, à celle d'une festivité relative, culminant parfois à une hadra qui peut réunir la totalité des présents qui, debout et en rangs serrés, se livrent à des exercices de respiration cycliques, rythmiques et très sonores en prononçant le nom de Dieu (Allah) accompagnés de mouvements corporels en balancier (balancement avant/arrière du buste). Durant cet exercice, appelé imāra, les chanteurs rivalisent d'imagination et de savoir-faire en improvisant mélodies et variations poétiques sur le thème de l'amour divin dans une joute vocale. Certains disciples gagnés par l'ivresse mystique et l'extase $(h \bar{a} l)$ fondent en larmes ou bondissent en criant le nom de Dieu.

L'espace profane est le lieu où la présence du divin est comme masquée par des activités ordinaires et triviales. Il s'agit de "l'avant-scène » (ou les coulisses) composée ici du vestibule, de la cuisine et de la salle de bains/toilettes. Il n'est pas nécessaire d'y revêtir la djellaba mais il est fermement interdit d'y être pieds nus au risque de ramener des impuretés dans l'espace rituel. Il est formellement interdit d'apporter dans les toilettes et la salle de bains les textes sacrés (Coran et recueil de litanies) ainsi que la subha (chapelet).

Le tableau suivant résume les actes rituels et les dispositifs présents dans la zāwiya:

\begin{tabular}{|c|c|c|}
\hline MARQUEURS & ESPACE RITUEL & ESPACE PROFANE \\
\hline $\begin{array}{l}\text { Actes } \\
\text { rituels }\end{array}$ & $\begin{array}{l}\text { - Prières canoniques collectives } \\
\text { et individuelles } \\
\text { - Baisemain rituel } \\
\text { - Réunion d'invocation } \\
\text { - Cantiques } \\
\text { - Enseignements } \\
\text { - Exercices corporels } \\
\text { - Repas collectifs, thé }\end{array}$ & $\begin{array}{l}\text { - Port de la djellaba } \\
\text { - Toilette rituelle } \\
\text { - Baisemain rituel } \\
\text { - Préparation des repas / } \\
\text { rafraîchissements / thé }\end{array}$ \\
\hline $\begin{array}{l}\text { Dispositifs } \\
\text { visuels }\end{array}$ & $\begin{array}{l}\text { - Portraits du cheikh } \\
\text { - Lettres du cheikh } \\
\text { - Photographie de la zâwiya-mère } \\
\text { (Maroc) } \\
\text { - Liste des règles de conduite } \\
\text { - Articles de presse } \\
\text { - Panneaux d'affichage }\end{array}$ & - Panneaux d'affichage \\
\hline
\end{tabular}


Plan de la zâwiya ${ }^{19}$

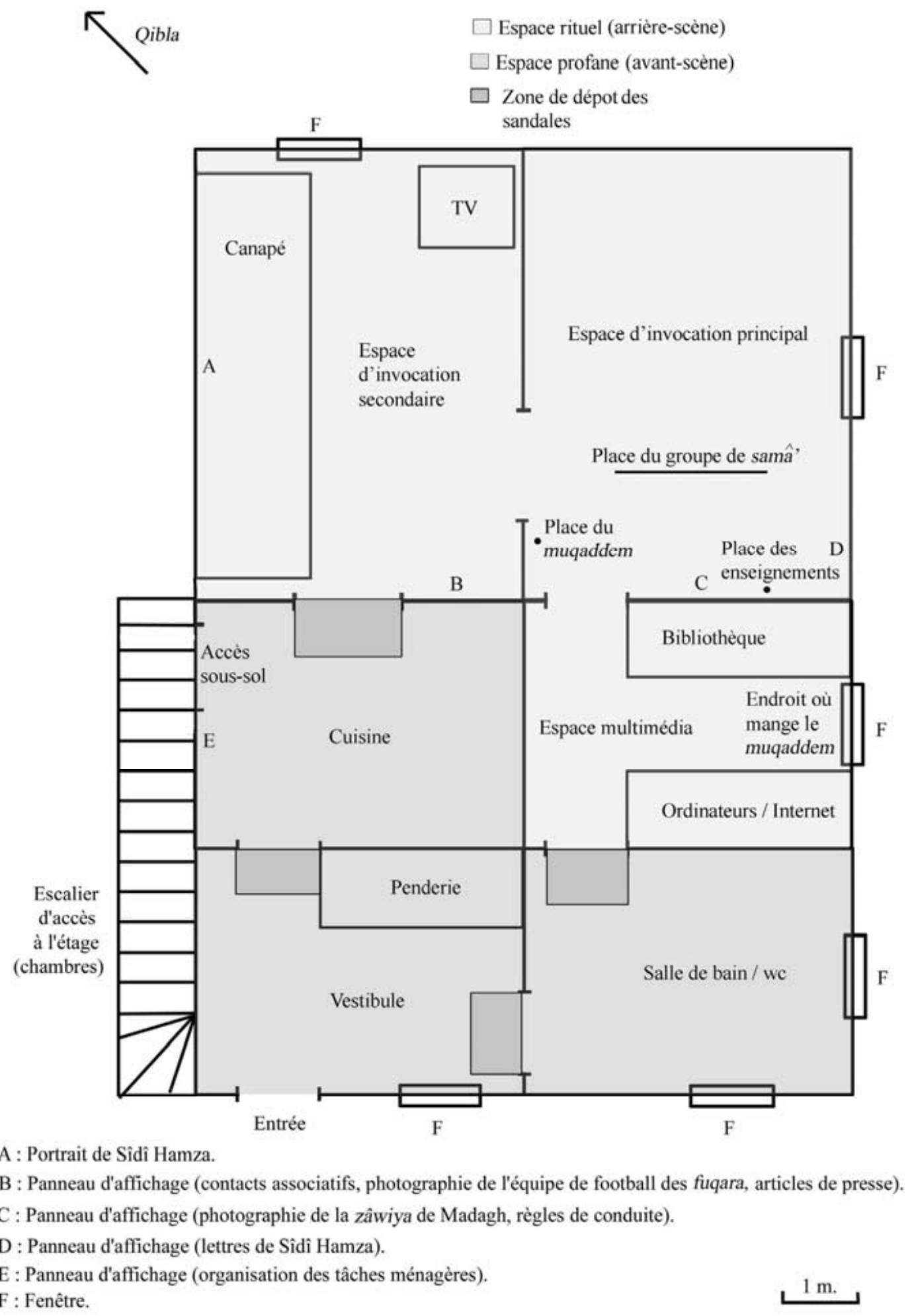

19. Plan relevé en février 2004. 
62 - Archives de SCIENCES SOCIALES Des Religions

La présence du divin dans l'espace domestique induit, pour les disciples, des règles comportementales précises établies par le shaykh pour codifier les relations entre les individus.

\section{La quête de l'" autoperfectionnement " et du savoir-vivre}

Dans ses études sur le salut personnel de l'individu, Max Weber (1998 [1909] : 545-567) oppose les activités cultuelles et rituelles à l' " autoperfectionnement » qui peut être de nature éthique ou mystique. Dans ce dernier cas, le salut est rattaché à un certain état d'illumination mystique ou d'union avec le divin, conséquence d'un agir comportemental conforme à des règles éthiques édictées par un référent d'autorité religieuse. Le respect des prescriptions comportementales doit permettre la réalisation d'un certain état d'être, à la fois garant et signe du salut. Dans la mystique, la dimension d'intériorité, de sentiment personnel, de paix intérieure, de joie et de quiétude est fondamentale. Il s'en dégage une véritable dévolution de son être comme de son existence terrestre qui doit être mise en relation perpétuelle avec le divin. Le but est ici, pour l'aspirant au cheminement initiatique, de pouvoir vivre une renaissance spirituelle, une évolution morale qui lui permette de se rapprocher du modèle prophétique de "l'homme parfait » (al-insān al-kāmil). Ainsi, pour F., 27 ans, étudiant, le soufisme a entraîné un changement d'environnement relationnel et social qu'il juge positif :

J'ai arrêté de faire des choses contraires à l'islam, comme boire de l'alcool. Cela a fait le vide autour de moi, je me suis séparé de certaines personnes, j'ai changé de fréquentations, les fuqarā' sont devenus mes meilleurs amis. Parallèlement, je me suis fiancé et j'ai un projet de vie. Le soufisme fait du ménage dans ta vie et te permet de devenir meilleur. Pour cela, il faut accepter de suivre les conseils du cheikh, car il est en contact avec des choses qu'on ne soupçonne pas. Il est divin. C'est une bénédiction pour nous d'être ici, d'être en contact avec les autres fuqarā'... Nous, les disciples, nous sommes plus qu'une famille.

Dans la méthode d'autoperfectionnement mystique existe donc une discipline assidue et permanente proche de la soumission aux règles établies par le cheikh. Cette doctrine forge un " habitus volontaire de l'émotion » (Gole, 2003 [1993] : 153) qui normalise et codifie chaque instant de la vie sociale, des émotions intimes au maintien corporel en passant par les comportements individuels et collectifs. Il semble que cette recherche d'un habitus volontaire de l'émotion, créatrice de liens intenses entre les croyants, fait la spécificité de la voie mystique d'autoperfectionnement - laquelle met l'accent sur l'amour, l'abnégation, la décence du comportement et de la parole, la retenue, l'humilité, la sagesse, la considération pour autrui et le rejet de l'agressivité - par rapport à toutes les diverses pratiques d'extase éphémères de types chamaniques ou extatiques. Suivre l'enseignement spirituel d'un cheikh, c'est véritablement accepter de se 
« civiliser» (au sens de Norbert Élias) ${ }^{20}$ par l'autocontrôle des pulsions et le rejet de l'agressivité. Accepter la méthode d'autoperfectionnement mystique c'est accepter de se conformer à ses prescriptions strictement codifiées définissant les actes de la vie quotidienne. Il en découle la mise en scène d'une certaine manière de concevoir le respect dû aux autres comme à soi-même, soit, au final, une convention de savoir-vivre en collectivité. Ce code de vie mondain impose et met en scène des règles comportementales, une tenue vestimentaire et du matériel rituel à chaque individu.

Tous les disciples présents dans la zāwiya doivent revêtir une tenue précise, posséder du matériel rituel et adopter un certain maintien physique qui est réglementé et définit par de nombreuses recommandations orales (le muqaddem et les fuqarā' se chargent d'expliquer - et de rappeler - comment adopter le «bon comportement » et une bonne tenue physique) et écrites (dans l'espace d'invocation principal, une affiche rédigée en français rappelle à tous les règles de conduite fondamentales). Ces recommandations sont :

Etre assis en tailleur, dos droit et en rang durant les invocations collectives (en cas de fatigue, se mettre debout sans abandonner les invocations), ne pas s'allonger, ne pas somnoler, éviter les va-et-vient, ne pas faire de gestes brusques, être en état de pureté rituelle, interdiction d'être pieds nus dans les espaces profanes (la salle de bain, la cuisine et les toilettes), obligation d'être pieds nus dans les espaces rituels (espaces d'invocations et espace multimédia/bibliothèque). Observer le silence (même pour les fuqarā' qui préparent le repas collectif), ponctualité (arriver parmi les premiers pour les invocations collectives), fermer les yeux durant les invocations collectives, bouger les lèvres pendant les lectures silencieuses du Coran et pendant les invocations mystiques, éviter les gestes brusques, les clins d'œil et les plaisanteries, adopter une attitude de pudeur vis-à-vis des autres fuqară', ne quitter l'assemblée qu'à la clôture des invocations collectives.

Les fuqarā' convertis peuvent, s'ils le souhaitent, changer leur prénom qui sera précédé, comme pour tous les autres, d'une marque de respect symbolisé par la formule « $S \bar{\imath} »$ ou « $S \bar{l} d \bar{\imath} »($ (Monsieur»). Cette formule de politesse agit encore comme un signe du lien social, un faqīr âgé ou ancien se doit d'appeler « $S \bar{\imath} d \bar{\imath} »$ même un tout jeune ou un nouveau faqī $r$, les adeptes aimant à rappeler que dans la pratique spirituelle, le «temps » n'existe pas. Un nouveau venu est immédiatement reconnu par ses frères comme un des leurs au même titre que les " anciens ". Mis à part cette adresse respectueuse, il existe un autre signe à double fonction, de gratitude et de salutation : le baisemain mutuel que les fuqarā

20. Cette expression est à comprendre dans le sens d'une transformation ou d'une modification du comportement de l'individu liée à l'autocontrôle de plus en plus accentué des pulsions et de l'auto-répression de l'agressivité. Dans La Dynamique de l'Occident (2003 [1939]), Élias avance que le " processus de civilisation » se diffusa dans la société occidentale par un processus d'imitation comportementale des couches sociales inférieures vis-à-vis des couches supérieures. Cependant, cette mission "civilisatrice " se retrouve dans la méthode d'autoperfectionnement mystique qui tend à « civiliser » les destinataires de son message. 
font soit lorsqu'ils se rencontrent soit après chaque prière collective : d'un seul geste, ils se serrent et s'embrassent mutuellement le dessus de la main droite, en prononçant «que la paix soit avec toi » (as-salām 'alaykum). À la fin de chaque cérémonie spirituelle, tous les fuqarā' présents à la zāwiya doivent donner ce baisemain rituel à la totalité des "frères ». La tenue vestimentaire des disciples s'insère dans un système de marqueurs qui a pour fonction de manifester et d'appuyer la position de la partie (le faqîr) par rapport au tout (la tarīqa). Appartenir à la tarīqa et visiter la zāwiya impliquent la possession nécessaire de matériel en relation étroite avec la pratique spirituelle. L'acteur étant soumis à l'attention continuelle de ses pairs, les marqueurs sociaux prennent ici la forme de signes. Ceux-ci se composent d'une djellaba ou d'une gandoura (djellaba sans capuche et sans manches), un signe culturel distinctif, et d'une subha (chapelet) pour le comptage des séries d'invocations. Fabriquée à la zāwiya-mère de Madagh, uniquement pour la tarīqa, celle-ci se compose de cent grains $(10+45+45)$, contrairement aux quatre-vingt dix-neuf grains des chapelets musulmans traditionnels. Elle est fournie au faqīr lors de la prise du "pacte " ('ahd) et de la communication de l'invocation individuelle (wird). La totalité du corpus liturgique utilisé par la tarīqa est conservée dans la bibliothèque et distribuée sous forme de fascicules lors des ijtima $\bar{a}^{\text {'s }}$ par les fuqară'-adjoints. La subha et ces textes font l'objet d'une attention particulière : ils ne doivent pas être déposés sur le sol de la zāwiya (mais, par exemple, sur des coussins) et en aucun cas être emportés dans les toilettes, espace désigné par les fuqarā’ comme étant "impur». Selon Goffman, ces obligations définissent la «façade personnelle » des acteurs, c'està-dire leur aspect extérieur composé des vêtements, des attitudes, de la manière de s'exprimer, des mimiques, des comportements et autres gestuelles. Celle-ci, adoptée par tous, devient une « façade collective » (Goffman, 1973:30-33) revêtue par les disciples dans le vestibule lors de leur arrivée sur les lieux. Elle leur sert à réaliser tous les actes qui se déroulent dans l'espace rituel.

Dessin de la «façade collective » des fuqarā' :

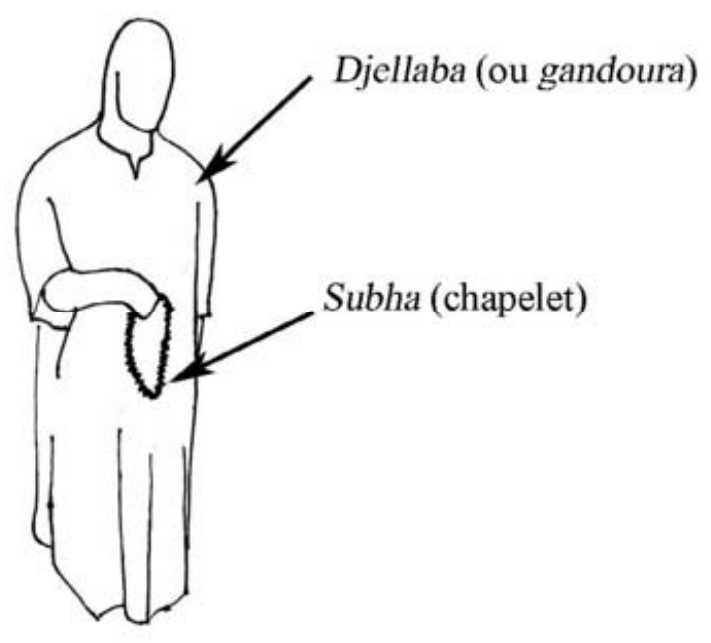


La position à adopter durant les invocations individuelles et collectives est assis en tailleur et le dos droit :

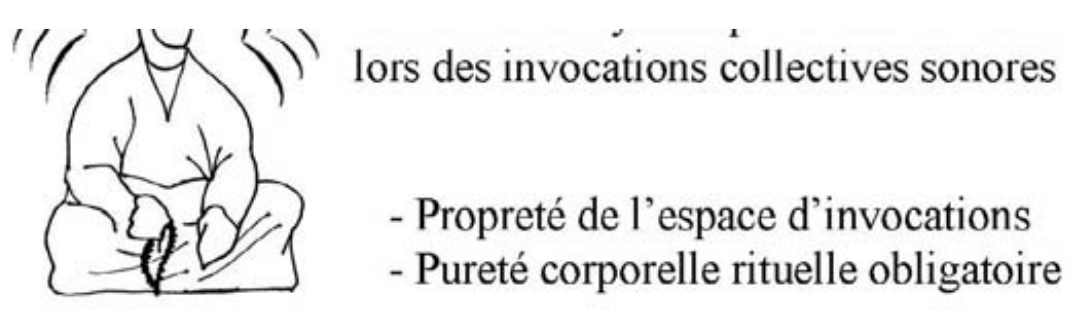

\section{Un sanctuaire secret}

En somme, la zāwiya Qâdiriyya-bûtchichiyya rencontrée en banlieue parisienne joue un rôle polyvalent lié à sa capacité à proposer un éventail de services qui, ailleurs, appartiennent à d'autres autorités : apprentissage religieux canonique, aspects du droit malékite, lectures collectives du Coran, enseignement de l'histoire du soufisme sunnite et de la vie du Prophète, cours de français, d'arabe classique et de théâtre, matchs de football, pratique de chants liturgiques, communication via Internet, organisation de conférences et de concerts spirituels. Cette palette d'activités offre au disciple la possibilité de s'engager dans un ordre religieux selon ses désirs et ses propres affinités. Au cours de sa présence dans ces lieux, le corps de l'adepte est modelé temporellement par un protocole normatif qui favorise un lien interpersonnel intense entre les fidèles. Articulant ainsi une forme de sociabilité qui met en situation des usages et des codes sociaux définissant le rôle du disciple et celui de sa hiérarchie, cette zāwiya permet aux affiliés la possibilité d'un usage pratique du divin en articulant une conduite sociale et morale conforme à un modèle d'homme, le cheikh fondateur de la tarĩqa.

Cette zāwiya française est aussi un sanctuaire secret (accès connu des seuls affiliés, absence d'indication sur la boîte aux lettres), sacralisé et fortement hiérarchisé. Les stratégies d'essaimage employées par les dirigeants de la confrérie depuis le Maroc la place dans une relation de subordination vis-à-vis de la zāwiya-mère marocaine. Cette dépendance, à la fois symbolique et structurelle, reste liée à la situation d'émigration de son jeune dirigeant, descendant du cheikh marocain, mandaté par lui pour entourer les jeunes disciples et organiser la promotion de la doctrine dans toute l'Europe. 
66 - Archives de sCiENCES SOCIALES DES RELigions

Il est toutefois regrettable, dans le cas étudié ici, que la confrérie Qâdiriyyabûtchichiyya se désintéresse de son environnement socioculturel immédiat et ne s'investisse en rien dans la cité. La zāwiya offre certes un espace de sociabilité, un réseau de solidarité réconfortant, mais à destination exclusive de ses disciples - principalement des jeunes, étudiants et sans emploi - en quête spirituelle ou fragilisés par leurs expériences antérieures.

Mehdi NABTI

EHESS CADIS

mehdinabti@hotmail.com

Agnouche Abdelatif, 1987, Histoire politique du Maroc : pouvoir, légitimités et institutions, Casablanca, Éditions Afrique Orient.

Amrous Mustafa, 1986, Les confréries religieuses et l'islamisme au Maroc au XIX et $X X^{e}$ siècles, Thèse de doctorat de troisième cycle de sociologie, Paris, Université Paris 10.

Astener Albert et Dassetto Felice, 1984, L'islam transplanté. Vie et organisation des minorités musulmanes de Belgique, Anvers, Epo.

BONTE Pierre, 2001, Émirs et présidents : figures de la parenté et du politique dans le monde arabe, Paris, CNRS Éditions.

BOUASRIA Abdelilah, 2007, "Sufism as a revolutionary discourse in Morocco? » in Revolutions: Revisited, Revised, Redesigned, Frankfurt, P. Lang.

Boubrik Rahal, 2000, «Fondateurs et héritiers. La gestion d'une succession confrérique (Mauritanie) », Cahiers d'Études Africaines, 159, pp. 433-465.

BEN Driss Karim, 2002, Sidi Hamza al Qâdirî Boudchich. Le renouveau du soufisme au Maroc, Beyrouth, Albouraq.

Berque Jaques, 1955, Structures sociales du Haut Atlas, Paris, PUF.

Crapanzano Vincent, 2000, Les Hamadcha. Une étude d'ethnopsychiatrie marocaine (The Hamadsha: A Study in Moroccan Ethnopsychiatry, 1973), Paris, Sanofi Synthélabo.

Dermenghem Émile, 1982 (1954), Le culte des saints dans l'islam maghrébin, Paris, Gallimard.

Dialmy Abdessamad, 2000, "L'islamisme marocain : entre intégration et révolution ", Archives des Sciences Sociales des Religions, 140, pp. 5-27.

DurKHeIM Émile, 1998 (1912), Les formes élémentaires de la vie religieuse : le système totémique en Australie, Paris, PUF.

Eickelman Dale, 1976, Moroccan Islam. Tradition and Society in a Pilgrimage Center, Austin, University of Texas Press.

-, 1981, "The study of Islam in local contexts ", Contribution of Asian Studies, 17, pp. 1-17.

Élias Norbert, 2003, La dynamique de l'Occident (Über den Prozeß der Zivilisation. Soziogenetische und psychogenetische Untersuchungen, 1939), Paris, Calmann-Lévy.

Elboudrari Hassan, 1985, La "Maison du cautionnement». Les Shurfa d'Ouezzane de la sainteté à la puissance : étude d'anthropologie religieuse et politique (Maroc: XVII ${ }^{e}$ $\mathrm{XX}^{e}$ siècles), Thèse de doctorat d'anthropologie, Paris, EHESS. 
Hammoudi Abdallah, 2001, Maîtres et disciples : genèse et fondements des pouvoirs autoritaires dans les sociétés arabes. Essai d'anthropologie politique, Paris, Maisonneuve et Larose.

GEERTZ Clifford, 1973, "Religion as a Cultural System », The Interpretation of Culture, New York, Basic Books, pp.87-125.

-, 1992, Observer l'Islam. Changements religieux au Maroc et en Indonésie (Islam Observed, Religious Development in Morocco and Indonesia, 1968), Paris, La Découverte.

-, 2002, Savoir local, savoir global: les lieux du savoir (Local Knowledge: Further Essays in Interpretive Anthropology, 1983), Paris, PUF.

Gellner Ernest, 1981, Muslim Society, London, Cambridge University Press.

-, 2003, Les Saints de l'Atlas (Saints of the Atlas, 1969), Paris, Bouchene.

Gole Nilufer, 2003 (1993), Musulmanes et modernes. Voile et civilisation en Turquie, Paris, La Découverte.

GofFMAn Ervin, 1973, La mise en en scène de la vie quotidienne. 1. Représentation de soi. 2. Les relations en public (The Presentation of Self in Everyday Life, 1959. Relations in Public: Microstudies of the Public Order, 1971), Alençon, Éditions de Minuit.

NABTI Mehdi, 2007, La confrérie des Ä̈ssâwa du Maroc en milieu urbain. Les pratiques rituelles et sociales du mysticisme contemporain, Thèse de doctorat de troisième cycle de socio-anthropologie, Paris, EHESS.

Trimingham Spencer, 1971, The Sufi Orders in Islam, London, New York, Oxford University Press.

Weber Max, 1998 (1909), Économie et société dans l'antiquité, Paris, La Découverte.

http://www.saveurs-soufies.com (site officiel de la zâwiya enquêtée).

GEOFFROY Éric, 2007, http://www.oumma.com/Le-soufisme-et-la-France-partie-1

-, http://www.oumma.com/Le-soufisme-et-la-France-partie-2 
68 - Archives de SCIENCES SOCIALES DES RELIGIONS

\section{Résumé}

Cet article décrit et analyse l'organisation et le fonctionnement d'une zāwiya française de la confrérie soufie marocaine Qâdiriyya-Bûdchichiyya. Les données de l'auteur proviennent de l'observation participante, d'entretiens libres et semi-directifs appliqués à une enquête de terrain à l'intérieur de l'une des zāwiya-s de cette confrérie située en banlieue parisienne. Par une approche microsociologique, le but est de saisir, d'une part, les modalités selon lesquelles une confrérie maghrébine contemporaine s'implante en France, et, d'autre part, ce que la doctrine mystique représente aux yeux d'adeptes vivant dans une société laïque de tradition catholique.

Mots-clés : soufisme, zâwiya, islam, France, Qâdiriyya-bûtchichiyya.

\section{Abstract}

This article is a microsociological study of a French zāwiya of the Moroccan soufi brotherhood Qâdiriyya-Bûdshishiyya. The data of the author come from the participating observation, of free and semi-directing interviews applied to an investigation inside one of the zāwiya-s of the brotherhood located in Parisian suburbs. The aim is to discover how a contemporary North-African brotherhood takes root in France and what the mystical doctrine represents for the disciples who lives in a secular society of catholic tradition.

Key words: Sufism, zawiya, Islam, France, Qâdiriyya-Bûtshishiyya.

\section{Resumen}

Este artículo describe y analiza la organización y el funcionamiento de una zâwiya francesa de la cofradía sufi marroqui Qâdiriyya-Bûdshishiyya. Los datos del autor provienen de la observación participante, de entrevistas abiertas y semi-dirigidas aplicadas a una investigación de campo de tres semanas (febrero de 2004) dentro de una de las zâwiya-s de esta cofradía situada en la periferia de Paris. Desde un enfoque microsociológico, el objetivo es comprender, por un lado, las modalidades a través de las cuales una cofradía magrebi contemporánea se implanta en Francia, y, por otro lado, lo que la doctrina mística representa a los ojos de adeptos que viven en una sociedad laica de tradición católica.

Palabras clave: sufismo, zâwiya, Islam, Francia, Qâdiriyya-Bûtshishiyya. 